\title{
VEGF-A Expression in Bladder Transitional Cell Carcinoma and Clinical Significance
}

\author{
Yong MA \\ Qiqihar Medical University \\ Qiqihar, China
}

\begin{abstract}
Detection of Vascular Endothelial Growth Factor A (VEGF-A) expression in bladder transitional cell carcinoma, it studies their impact on the micro-vessel density (MVD), and in patients with transitional cell carcinoma of the age, gender, tumor grade, clinical staging and prognosis, so as to explore the bladder muscle VEGF-A in tumor angiogenesis that plays a role in its clinical significance.
\end{abstract}

Keywords:VEGF-A; Cell Carcinoma; Meaning.

\section{INTRODUCTION}

Bladder cancer is the most common malignancy of the urinary system, regardless of its incidence or mortality rate ranks first in urological tumors. Bladder cancer histological types are divided into bladder transitional cell carcinoma, squamous cell carcinoma of the bladder, and bladder cancer. Transitional cell carcinoma accounts for about $90 \%$. Studies have shown that the incidence of bladder cancer is increasing year by year, serious harm to people's health. Therefore, for bladder cancer, especially bladder transitional cell carcinoma study makes sense.

BTCC exact pathogenesis has not yet been fully elucidated. In recent years, extensive research on the molecular biology of tumor pathogenesis of multiple genes in cancer cells synergistic theory is now widely accepted by people. From the simple role of oncogenes and tumor suppressor genes, the human body, like any activation or inactivation of a tumor suppressor gene in cancer gene, may lead to cancer. In fact, the incidence of cancer is an extremely complex process of malignant cells, and its development is not just an individual or the result of several factors, but a number of factors, multiple genes, multiple stages of the comprehensive development process, both intrinsic genetic factors, there are external environmental factors, which is the result of interaction between the two. Its intrinsic molecular biological mechanisms and relevance are not very clear, which remains to be further research scholars. Oncogenes and tumor suppressor genes, respectively, cell growth bear positive and negative aspects of regulation is an important factor in maintaining normal cell homeostasis. It is an inherent part of the genome of normal cells. Our study of cancer-promoting genes VEGF, hoping mechanism from the perspective of molecular biology can be more in-depth understanding of tumor genesis, and recognizes VEGF in bladder transitional cell carcinoma of the role, to provide more theoretical basis for clinical work.

\author{
Hongkun HAN \\ The First Affiliated Hospital of Qiqihar Medical \\ University \\ Qiqihar, China
}

VEGF (vascular endothelial growth factor), also known as vascular permeability factor, is stellate cells, such as from 1989 Gosper proliferation of cultured bovine pituitary follicular fluid separated a specific antigenic endothelial cell mitogenic factors, Then it is the amino acid sequence of vascular permeability factor is the same, it will be seen that they are a class of substances. VEGF is the only way to know the specific role of the current generation factor in vascular endothelial cells, VEGF endothelial cells by binding to receptors on each other, causing a series of signal transduction. This process stimulates the value of vascular endothelial cells that induced angiogenesis, which regulates vascular permeability and to promote the formation of blood clots, tumor formation, transfer of prerequisites, and in the growth and metastasis plays an important role. Currently, it is found in highly expressed in many cancers VEGF mRNA tissue and cultured cell lines. Such as: colon, breast, lung, kidney, etc., but in bladder transitional cell carcinoma study reported in the country is very small.

VEGF exerts its biological effects, which must combine with receptors can be achieved in the future. VEGF VEGFR usually has a 1, VEGFR a 2, NP a 1, NP-2 and other receptors. VEGF receptor binding and different will produce different biological effects. The VEGFR-1 and VEGFrelated inhibition of endothelial function biological effects are related to the contrary, VEGFR-2 is a major regulator of endothelial cell signaling pathways and the majority of the known VEGF, etc., such as reproductive cells, migration and penetration. Studies have shown that the occurrence of these two receptors are crucial for the formation of blood vessels, the lack of any one receptor gene expression in experimental animals, and have appeared in embryonic vascular dysfunction and death.

\section{BIOLOGICAL FUNCTION OF VEGF}

Malignant tumor growth and metastasis must rely on the rich supply of nutrients, blood vessel tissue generated as nutrients and oxygen into the tumor tissue, the tissue metabolites out of the extracellular and migration of tumor cells are to target organs laid an important foundation conditions. If there is no angiogenesis, tumor tissue will be in vitro production! Monzonite volume of less than $4 \mathrm{~mm} 3$, in vivo tumor does not exceed $1-2 \mathrm{~mm} 3$. So Angiogenesis is essential for tumor growth and metastasis conditions, and tumor genesis, development and metastasis are closely related. Understanding of tumor angiogenesis of cancer occurrence, development, invasion and metastasis of 
biological characteristics and mechanism as well as antiangiogenesis therapy has important theoretical and clinical applications. Angiogenesis process includes the following steps:

Endothelial cell activation, angiogenic phenotype formation: vascular basement membrane and extracellular matrix degradation by proteases; endothelial cell proliferation, migration, tube formation: the formation of new blood vessels lumen in communication networks. This process is largely induced by angiogenic factors and the regulation of angiogenesis inhibitors; angiogenesis inhibitors reduce the secretion and blood vessels to induce cytokine secretion is increased, leading to angiogenesis. Recently, researchers found that tumor angiogenesis because of the more than 30 species, such as vascular endothelial growth factor, angiostatin, endostatin, fibrinogen self-growth factors, including vascular endothelial growth factor and its receptor could be specifically promoted cell division, proliferation and migration, which plays a vital role in the process of tumor angiogenesis.

VEGF promotes tumor $\mathrm{J}$ (ii mechanism of angiogenesis is: VEGF enhanced vascular permeability, blood vessel caused mainly fibrinogen protein extravasation since the original, the original self-protein fibers deposited in the capillary matrix for tumor cells the establishment and growth of new blood capillaries network to provide the best matrix; VEGF receptor binding. It is to play its specific activity of endothelial cell mitogen stimulated proliferation of vascular endothelial cells, thereby contributing to the establishment of the blood vessel formation and vascular network, provide adequate nutrients for the growth of the tumor.

\section{A. Promote vascular endothelial cell proliferation}

VEGF as endothelial-specific cytokine can stimulate cultured endothelial cells in mitosis and migration, as well as the formation of blood vessels in vivo. Currently, it has already promote endothelial cell proliferation mainly VEGFA121 and VEGF-A1650 research process for renal tumors found, VEGF expression and its receptor are promoting endothelial cell mitosis, thus contributing to nascent tumor blood vessels. It cannot rule out other cytokines on endothelial expression, but you can explain certain VEGF angiogenic endothelial cell proliferation. VEGF can selectively enhance endothelial cell mitosis, in vitro endothelial cell growth promotion, promotion of angiogenesis in vivo. During the growth of tumor cells, secreted VEGF is considered one of the most important factors to stimulate endothelial cell proliferation. Therefore, blocking the secretion and function of this factor would be an important means of inhibiting angiogenesis. It should further study the interaction of VEGF vascular endothelial cell proliferation and other cytokines may cause to promote.

\section{B. Increase in vascular permeability}

VEGF vascular penetrant as the strongest can increase vascular permeability, especially micro vascular permeability, causing leakage of plasma proteins into the extracellular matrix, calm in the extracellular matrix of fibroblasts and endothelial provide the conditions to move into the cell matrix, providing nourishment for the establishment of tumor cell growth and new capillary network.

\section{Support to promote the generation of blood vessels}

VEGF stimulates endothelial cells to produce proteases, such as interstitial collagenase and matrix protein degradation, so that the matrix disintegration, VEGF releases, beneficial blood vessel formation. VEGF also make endothelial gene activation. It can induce endothelial cells to express urokinase-type plasminogen activator, tissue-type plasminogen activator and urokinase-type plasminogen activator receptors, thus contributing to penetrate into the plasma protein extravascular plasma protein forming region clots, as angiogenesis support. Baker and other mark-taking by ELISA, VEGF and PAS component colorectal cancer tissue and normal tissue found uPA, uPAR, plasminogen activator inhibitor (PAI-1) and VEGF were significantly rising in tumor tissue high, which also observed VEGF and uPA, uPAR, PAI-1 relationship between the stage and the Dukes are closely related.

\section{Inhibition of tumor cell apoptosis}

Experiments have proved that proliferation and VEGF positive control group that was not statistically significant tumor cells, but the number of apoptotic cells significantly decreased, and NOS activity strengthened. The neuroblastoma cells were placed in standard medium and VEGF in the medium was added after 24 hours incubation, with reduction of serum $\mathrm{TNF}-\mathrm{a}$ and nutrients to induce apoptosis, and it found that the expression of BCL-2 cells increased VEGF plus 33, and effectively inhibition of TNF-a and it can reduce nutrition on serum tumor induced apoptosis of neuroblastoma.

\section{E. It can significantly affect immune function}

Oyama found that tumor cells secrete VEGF can inhibit hematopoietic progenitor cell core transcription factor NF$\mathrm{KB}$ activation, induction of dendritic cells (dendritic cell, DC) precursor cells undergo apoptosis, thereby preventing DC maturation. DC cannot be induced to mature and / or lack of antigen-presenting function, resulting in immune function in patients with low tumor cells to escape immune surveillance body tumors, causing tumor cell proliferation and metastasis.

\section{VEGF RELATIONSHIP WITH CELL TUMORS}

VEGF in human normal tissue and tumor tissue is widespread, but in a variety of malignant tumors such as lung cancer, breast cancer, gastrointestinal cancer, ovarian cancer, renal cancer and other tissues showed high expression. The study found that both lung cancer cells in vitro, or in athymic nude mice inoculated with tumor cells to form tumors in nude mice bearing tumor cells in an animal model, or is highly expressed in human cancer tissues that are swollen VEGF. Studies using immunohistochemical staining of VEGF and micro-vessel density (MVD) in relation Saito found that, VEGF expression was significantly higher MVD of gastric carcinoma with negative expression of VEGF and VEGF positive blood group significantly increased chance of metastasis. VEGF and mutant p53 
protein is highly expressed in gastric cancer; vascular invasion and tumor micro-vessel density range were positively correlated. Other applications by ELISA in 37 patients with primary patients with gastric cancer and 10 cases of hair serum and plasma VEGF levels measured in healthy results showed that gastric cancer patients were significantly higher than normal levels of plasma VEGF, VEGF levels. The primary tumor size, vascular invasion, histological type, lymph node involvement are closely related, it has nothing to do with gender, age, lesion location and other factors. Fujisaki and other highly sensitive ELISA assay with colorectal cancer serum VEGF levels, the result was significantly higher than the control group, and significantly correlated with Dukes stage and serum CEA. Anthony et al [36j detected by in situ hybridization 46 cases of breast cancer biopsies and found that VEGF expression in tumor cells and tissue differentiation, lymph node metastasis, distant metastasis and early death 1\}: closely associated with breast cancer MVD was significantly correlation.

\section{EXPRESSION AND SIGNIFICANCE OF CANCER- PROMOTING FACTOR VEGF-A BLADDER TRANSITIONAL CEll CARCINOMA}

Bladder transitional cell carcinoma is the most common malignancy of the urinary system; it is also typical of angiogenesis active organ. As we all know, Oxygen and blood vessels are to provide nutrients to the tumor, so that the new generation of blood vessels in tumor growth, progression and metastasis. The process plays an important role, as well tumor invasion and metastasis strong source. We must also recognize that the formation of new blood vessels, which is to promote blood vessel growth factor closely related, and only highly active vascular-related factors, it is more favorable supply and generation of tumor blood vessel growth. Wang found higher VEGF expression and micro-vessel density of bladder cancer associated instructions cancer-promoting factor (VEGF) plays a positive role in the regulation of angiogenesis in the process of bladder cancer, VEGF is one of the major angiogenic factor. Angiogenesis is divided into the following steps: First, the endothelial cells are activated, formed angiogenic phenotype; followed by an outer vessel basement membrane degradation and extracellular matrix proteases action; Third, proliferation and migration of endothelial cells, neonatal vascular lumen formation; the fourth is new blood vessels into the lumen in communication network structure, with a lot of anastomosis. We know, from the blood vessel and the mammalian cell, it is less than 200um, if it exceeds this limit, oxygen and nutrients are not readily diffuse into cells, it is also not conducive to cell survival. Meanwhile, the growth of tumor cells also requires adequate blood supply. In theory, it can promote into tumor angiogenesis that will be able to promote the growth and metastasis of tumor cells, inhibition of tumor angiogenesis can inhibit the growth and metastasis of tumor cells. If in hypoxic tumor tissue and blood supply is not sufficient, there may be a higher degree of malignancy resistant hypoxic tumor cells. Therefore, that in bladder tumorigenesis, development and metastasis, VEGF has a very important role. The study proved that VEGF is the most important known vascular endothelial growth factor; VEGFspecific and role-play in all of pro-angiogenic factors VEGF expression in the strongest by proto-oncogenes and tumor suppressor gene of expression products. The dual control by inducing VEGF some proteolytic enzymes, endothelial cell proliferation, migration, increased neovascularization

There are construction, formation, and development of tumor-related pathology. VEGF also increases the permeability of tiny veins that fibrin and plasma protein extravasation, causing changes in the extracellular matrix of cells, cellulose-like deposition and formation of a new matrix for bladder cancer provides invasion and metastasis source of strength. Salven other detection in serum and urine of cancer patients in part to the increase in VEGF, Jacobsen et al reported by ELISA VEGF also elevated in the serum and urine of tumor, its level is associated with tumor stage.

\section{CONCLUSIONS}

VEGF-A expression in transitional cell carcinoma of the bladder muscle was significantly higher than in normal bladder muscle tissue, both highly statistically significant $(\mathrm{P}$ $<0.01)$ compared. VEGF-A expression and the relationship between the clinical and pathological factors prompted with increasing tumor grade and stage, the expression of VEGF-A was also significantly increased, which can help promote cancer development role.

\section{ACKNOWLEDGEMENTS:}

Heilongjiang Provincial Education Department Science and Technology Research Project (No.12521637)

\section{References}

[1] Zhang Liang. Angiopoietin -2 (Ang-2) and vascular endothelial growth factor (VEGF) expression and tumor angiogenesis in bladder transitional cell carcinoma and clinical significance of the relationship between [D]. Fujian Medical University, 2005.

[2] Jiang Hua, Chen Hong, Wang Weidong, Hangzhou Xia, Zhang Rongliang, Jianghao Wen, Ding Qian. The expression and clinical significance of $\mathrm{g}$ PEDF and VEGF in bladder transitional cell carcinoma [J] Jiangsu Medicine, 2010,01: 15-17.

[3] Yi Rui. MMP-9, VEGF and COX-2 expression in bladder transitional cell carcinoma and its significance [D]. Nanchang University, 2007.

[4] Liang Hao. P27, VEGF expression and significance in bladder transitional cell carcinoma of the research [D]. Henan University of Science and Technology, 2011.

[5] Feng Zhongling, Jiao Chuanchu, Ren Changshan, Li Yaling. Vascular endothelial growth factor (VEGF) receptor (Flk-1) expression in bladder transitional cell carcinoma [J] Chinese Journal of Clinical Oncology, 2001,02: 49- 51.

[6] Luo Chunli, Wu Hou, Bao Yixi, LI Yuanyuan. Vascular endothelial growth factor, cytokeratin 20 expression in bladder transitional cell carcinoma and its clinical significance [J] Chongqing Medical University, 2002,03: 304-306 